\title{
Dealing with correlated choices: How a spin-glass model can help political parties select their policies
}

\author{
M. A. Moore ${ }^{1}$ and Helmut G. Katzgraber ${ }^{2,3,4}$ \\ ${ }^{1}$ School of Physics and Astronomy, University of Manchester, Manchester M13 9PL, UK \\ ${ }^{2}$ Department of Physics and Astronomy, Texas A\&M University, College Station, Texas 77843-4242, USA \\ ${ }^{3}$ Materials Science and Engineering, Texas A\&M University, College Station, Texas 77843, USA \\ ${ }^{4}$ Santa Fe Institute, 1399 Hyde Park Road, Santa Fe, New Mexico 87501
}

(Dated: October 13, 2014)

\begin{abstract}
Starting from preferences on $N$ proposed policies obtained via questionnaires from a sample of the electorate, an Ising spin-glass model in a field can be constructed from which a political party could find the subset of the proposed policies which would maximize its appeal, form a coherent choice in the eyes of the electorate, and have maximum overlap with the party's existing policies. We illustrate the application of the procedure by simulations of a spin glass in a random field on scale-free networks.
\end{abstract}

\section{INTRODUCTION}

The application of statistical physics models to sociophysics problems has led to interesting studies, e.g., how coalitions form and how fragmentation can affect groups [14]. Here, we study how a political party might choose its policies to maximize its appeal to the electorate and produce a set of policies which have some "coherence" in the eyes of the voters and overlap with its existing policies. Suppose the party has produced a set of policies which it is considering for its manifesto. Usually, for the purposes of inclusion in its manifesto, the description of the policy is reduced to a short paragraph. News organizations reduce them further to onesentence statements. Examples of such reductions together with links to the full party manifestos can be found, for example, on the BBC website [5] for the UK 2010 General Election and those for just one topic, Crime, are laid out in the Appendix to this paper. The next step would be to put these short propositions via a questionnaire to a group of $M$ individuals who together are a representative sample of the entire electorate. They are to be asked whether on, e.g., a five-point scale they strongly agree or strongly disagree with the proposed policy. We shall label the $N$ propositions by $i=1,2$, $\ldots, N$, and we shall label the individuals of the group with $\mu$, where $\mu=1,2, \ldots, M$. If on the $i$ th proposition the $\mu$ th member of the group agrees strongly with it, it is associated with a response variable $R_{i \mu}=1$, strongly disagree $R_{i \mu}=-1$, and the three points on the scale between these two are given the values $1 / 2,0$, and $-1 / 2$, respectively. The average $m_{i}^{(0)}$ is then obtained for each issue:

$$
m_{i}^{(0)}=\frac{1}{M} \sum_{\mu=1}^{M} R_{i \mu}
$$

Should the $\mu$ th member of the group not respond to the $i$ th question, the average can be calculated setting their $R_{i \mu}=0$.

A value for $m_{i}^{(0)}$ close to \pm 1 defines a valence issue [6]. An example of a valence issue would be contained in the proposition that "The present high unemployment level is a terrible waste of human potential and must be brought down." Nearly everyone would agree with such a proposition. However, spe- cific proposals to reduce high unemployment are likely to be contentious and thus have values of $\left|m_{i}^{(0)}\right| \ll 1$. It is only policy proposals for which $\left|m_{i}^{(0)}\right| \ll 1$ which will be discussed here.

Modern elections are actually largely fought on valence issues and the perceived competence of parties and their leaders to deal with these [6-8]. Thus if unemployment is high, the opposition parties would simply use that fact as a stick to beat the ruling party. They will try to persuade the electorate that the existence of high unemployment shows that the government is either uncaring or incompetent, or both. Their own specific policies to deal with the problem will feature less in their campaign, as they are likely to elicit less than total support than the proposition that something should be done about high unemployment. While a party's actual policies may not be critical in determining the outcome of the election, they perhaps deserve attention from the electorate, as the successful party will, once in government, introduce many of the measures which were in its manifesto.

Unavoidably, there will be correlations between the responses to the various policies. Thus an individual member of the group who strongly supports the Conservative Party proposal (see the Appendix) to, e.g., "Strengthen stop and search powers to tackle knife crime" would be likely to support the proposition to "Reduce paperwork needed for stop and search procedures," although logically these are distinct proposals. We label the correlation between issues by $C_{i j}^{(0)}$ and measure it by calculating

$$
C_{i j}^{(0)}=\frac{1}{M} \sum_{\mu=1}^{M}\left(R_{i \mu}-m_{i}^{(0)}\right)\left(R_{j \mu}-m_{j}^{(0)}\right) .
$$

In addition, we define a variance $\Delta_{i}$ via

$$
\Delta_{i}=\frac{1}{M} \sum_{\mu=1}^{M}\left(R_{i \mu}-m_{i}^{(0)}\right)^{2} .
$$

Should it turn out that for some $i$ and $j,\left|C_{i j}^{(0)}\right| \approx \Delta_{i}$, one of them should be struck from the list as they are just being perceived as identical propositions (if $C_{i j}^{(0)} \approx \Delta_{i}$ ) or one is just the negation of the other (if $C_{i j}^{(0)} \approx-\Delta_{i}$ ). 
A party then has to decide which policy it should adopt bearing in mind the responses of the group. The purpose of this paper is to provide an effective procedure for doing this. If the party adopts policy $i$, we identify that choice with the "spin" variable $S_{i}=+1$, and if the party rejects that policy, $S_{i}=-1$. Only those policies $i$ with positive $S_{i}$ will be published in the party's manifesto. The simplest algorithm for choosing policies (i.e., the orientation of the spins) is to set

$$
S_{i}=\operatorname{sign}\left(m_{i}^{(0)}\right) .
$$

However, because of the existence of correlations, better choices are possible, as we shall see below. Parties will naturally wish to have policies which have coherence: Equation (4) simply ignores the correlations which exist between policies that are encoded in $C_{i j}^{(0)}$.

Furthermore, a party which has been in existence for some time will already have policies related to some of the issues in the list. It would prefer to have its new policies consistent with its existing policies. The procedure advocated in this paper can help in reconciling them.

The paper is structured as follows. We shall introduce in Sec. II a spin-glass model to develop an alternate algorithm to that of Eq. (4) which allows for the correlations between issues to be taken into account. Readers uninterested in the details should regard the spin-glass model as just a way of allowing for the effects of correlations. The spin-glass model provides a way of choosing a portfolio of policies which will be more coherent than that which would be provided by use of Eq. (4) and which could have better overlap with existing policies. In Sec. III we outline some of the relevant properties of spin glasses, illustrated by a synthetic data set from simulation results on an Ising spin glass in a random field on a scale-free network, and we conclude in Sec. IV with a discussion of other possible uses of the spin-glass approach to politics, as well as extensions to other problems where correlations between choices might be relevant.

\section{SETTING UP THE SPIN-GLASS HAMILTONIAN}

The Ising spin-glass Hamiltonian in a field is generically of the form

$$
\mathcal{H}=-\sum_{i<j}^{N} J_{i j} S_{i} S_{j}-\sum_{i=1}^{N} h_{i} S_{i}, \quad S_{i}= \pm 1 .
$$

We shall determine the interactions $J_{i j}$ and the fields $h_{i}$ for our problem by relating them to the $m_{i}^{(0)}$ and the correlations $C_{i j}^{(0)}$. In the high-temperature $T$ limit, $\beta=1 / T$ is small. Then, to leading order in $\beta$ :

$$
m_{i} \equiv\left\langle S_{i}\right\rangle \approx \beta h_{i}
$$

Similarly, the leading order expression for the cumulant correlation is

$$
C_{i j} \equiv\left\langle\left(S_{i}-\left\langle S_{i}\right\rangle\right)\left(S_{j}-\left\langle S_{j}\right\rangle\right)\right\rangle \approx \beta J_{i j} .
$$

We shall fix $\beta h_{i}$ by setting

$$
\beta h_{i}=m_{i}^{(0)}
$$

and $\beta J_{i j}$ by setting

$$
\beta J_{i j}=C_{i j}^{(0)} .
$$

It then only remains to fix $\beta$ itself, and this is done by arbitrarily making the variance of $J_{i j}$ unity. One can set $J_{i i}=0$ as self-correlations can play no role for Ising spins. Notice that we can make use of the high-temperature approximation for $m_{i}$ in Eq. (6) because we have assumed that all the $\left|m_{i}^{(0)}\right| \ll 1$; that is, there are no valence issues in the list. Some of the $J_{i j}$ will be positive and some negative. This would arise if the group is exposed to a wide range of propositions, (and would certainly happen if the proposals from all three parties were put together into one single questionnaire).

The basic properties of this Hamiltonian are well known [9]. As the temperature $T$ is reduced, a phase transition to a spin-glass state occurs even in the presence of the fields $h_{i}$ [9]. The values of $N$ relevant for us are probably of the order of $N \sim 500$. In Ref. [5] under 17 headings there were a total of 523 policies listed for the three parties, although some overlapped. Investigating the behavior of a system of approximately 500 spins is computationally nontrivial and would have to be done via a large-scale Monte Carlo simulation. At high temperatures the spins flip frequently, but as the temperature is reduced the system becomes "glassy," i.e., relaxation times grow and flipping occurs infrequently. In the limit when $T \rightarrow 0$, the system settles into its ground state configuration where the spins take the values $S_{i}^{(T=0)}$. It is proposed that the algorithm to choose which policies $i$ should be adopted is to select those for which

$$
S_{i}^{(T=0)}=1 .
$$

Note that in Sec. III we generalize this proposal to choosing one of the "pure" states of the spin glass, if this allows better consistency with a party's existing policies.

The advantage of this algorithm over the trivial one of Eq. (4) is that it allows for the interactions $J_{i j}$ which exist between the policies (i.e., the "spins"). Equation (4) ignores them entirely. The two outcomes can be very different in practice as the synthetic data studied in Sec. III show.

In the (Metropolis) Monte Carlo algorithm [10, 11] one focuses on the energy $\Delta E$ to flip a single spin. One has $\Delta E_{i}=2 H_{i}$, where the local field $H_{i}$ on the $i$ th spin is

$$
H_{i}=\sum_{j} J_{i j} S_{j}+h_{i}
$$

One picks one of the $N$ spins at random and if the energy is decreased by flipping the $i$ th spin (i.e., if $\Delta E_{i} \leq 0$ ), then that spin is flipped; if it is increased, it is flipped with a probability $\exp \left(-\beta \Delta E_{i}\right)$. If one does this enough times, one generates an equilibrium ensemble at the inverse temperature $\beta$ from which thermodynamic averages can then be calculated. Notice that all the thermodynamical properties depend on the choice of 
both the $J_{i j}$ and the $h_{i}$. At high temperatures $(\beta \rightarrow 0)$ it is the fields $h_{i}$ which dominate the behavior. At $T=0$ the influence of the $J_{i j}$ is at its strongest. This is why the algorithm of Eq. (10) produces a portfolio of policies which incorporates the correlations $J_{i j}$ between them.

We shall in the next section detail some of the properties of spin glasses which have relevance to the problem of policy choice, using as an illustration simulations of a spin-glass model defined on a scale-free network with random values of $J_{i j}$ drawn from a Normal distribution with zero mean and standard deviation 1 , as well as $h_{i}$ drawn from a Normal distribution with zero mean and a standard deviation $H=0.1$. While parties do apparently use focus groups and the like for investigating the likely appeal of particular policies, we know of no publicly available data obtained from a large group which we could use. It is our hope that our algorithm will stimulate the release or production of such data for further analysis.

\section{PROPERTIES OF THE SPIN-GLASS MODEL}

The interactions $J_{i j}$ do not exist between all pairs $i$ and $j$; if the interactions are at the level of noise, that is, of $\mathcal{O}(1 / \sqrt{M})$, one should set $J_{i j}=0$ to speed up the simulation. However, some policies $i$ might have an interaction with many other policies $j$, while others may interact with only a few others. The situation, where there is a range in the number of spins with which a given spin interacts, has not been studied much in the spin-glass literature, although there is some work on scale-free networks [12-14] which can have a wide range in the number of spins to which a spin might be coupled. Because we do not know of real data relevant to test our proposed algorithm, we shall study a spin-glass model on a scale-free network as a proxy for real data. It would of course be useful to know if real data were well represented by a scale-free network. However, our procedure does not depend on the given network topology, as pure states and other spin-glass features exist also for networks of fixed connectivity.

Scale-free networks have edge degrees distributed according to a power law $\lambda$, with the probability $\mathcal{P}_{k}$ for a node (spin) to have $k$ neighbors being

$$
\mathcal{P}_{k} \propto k^{-\lambda}
$$

Typical networks are shown in Fig. 1: While few spins (i.e., political issues) have many interactions $J_{i j}$ connecting them to other spins (issues), many spins are connected to only a few other spins; the distribution follows the power law of Eq. (12). It could be that a few issues have extensive links to most other issues. For example, one of these issues could be related to the current state of the economy. The case where a few issues are dominant can thus be modeled by choosing a small value of $\lambda$, whereas when no issue in particular is strongly dominant, a large value of $\lambda$ can be used.

The graph-generation technique used in our simulation is discussed in detail in Ref. [13]. An upper bound is imposed on the allowed edge degrees of $k_{\max }=\sqrt{N}$, as well as a lower bound $k_{\min }=3$. Nodes with $k=0,1,2$ might exist with real data: Nodes with $k=0$ correspond to isolated spins, while spins at nodes with $k=1$ and 2 can be traced out modifying the coupling and fields for the remaining spins. Because we are studying synthetic data, rather than real data, we decided to suppress such nodes.

The spin-glass model generated by the procedure described in Sec. II is a model for which the mean-field ideas described in Ref. [9] are entirely appropriate. Such models have a rich and strikingly complicated behavior, primarily because of the existence of many pure states [17] in the thermodynamic limit $(N \rightarrow \infty)$. For Ising ferromagnets in zero field (i.e., when $J_{i j}=1 \forall i, j$ ) there are only two (pure) states, those with up $\left(S_{i}=1 \forall i\right)$ and down $\left(S_{i}=-1 \forall i\right)$ magnetization. However, spin glasses can have many pure states when $N$ is large. The pure spin states have a hierarchical relation to each other, called an ultrametric topology [9], which means that there are deep relationships between them.

In a situation where $N \sim 500$, the number of pure states is expected to be small [18]. The actual number of such pure states depends on the particular realization of the interactions $J_{i j}$ and the values of $h_{i}$. In a simulation at finite temperature, the system will settle into one of the pure states for long periods of time; i.e., it will be "stuck" close to a particular configuration of the $S_{i}$. Note that the ground state is always a pure state. It becomes progressively harder to do simulations as the temperature approaches zero. The easiest way to demonstrate the existence of pure states is to simulate two copies of the system of spins and monitor the overlap

$$
q=\frac{1}{N} \sum_{i=1}^{N} S_{i}^{(1)} S_{i}^{(2)}
$$

where $S_{i}^{(1)}$ denotes a spin in the first copy and $S_{i}^{(2)}$ denotes a spin in the second copy with the same interactions and fields. One can then determine the probability distribution $P(q)$ of the overlap, and the results of doing this are shown in Fig. 2 for 6 realizations of the $J_{i j}$ and $h_{i}$. The number of peaks (features) in $P(q)$ is equal to the number of pure states [18]. At high temperature there is but one peak (not shown), but as the temperature is reduced, more peaks appear, often as shoulders on existing peaks. When several pure states are present, each of the copies will be in one of them at low temperatures, and the value of their overlap will be a peak in $P(q)$. Shoulders evolve into two separate peaks as the temperature is reduced. From a study of 29 randomly chosen samples for $N=512$, we estimate that the average number of peaks/features at the lowest temperature we could simulate $\left(T=0.2862 \ll T_{c}\right)$ $[13,14])$ was for $\lambda=2.5,6.27 \pm 0.70$ at $H=0$ and $3.83 \pm 0.41$ at $H=0.1$, while for $\lambda=4.5$, the number of peaks/features was on average $5.65 \pm 0.52$ at $H=0$ and $3.96 \pm 0.39$ at $H=0.1$ [19]. The statistical error bars are computed using a bootstrap analysis. A systematic study of the number of states as a function of $N, H$, and $\lambda$ would be valuable but unfortunately very time consuming. What is striking to us is the variability in the number of pure states from sample to sample, but despite that there seems to be a robustness about the data. Figure 1 shows that there are large differences in the connectivity of the scale-free networks for $\lambda=2.5$ and 

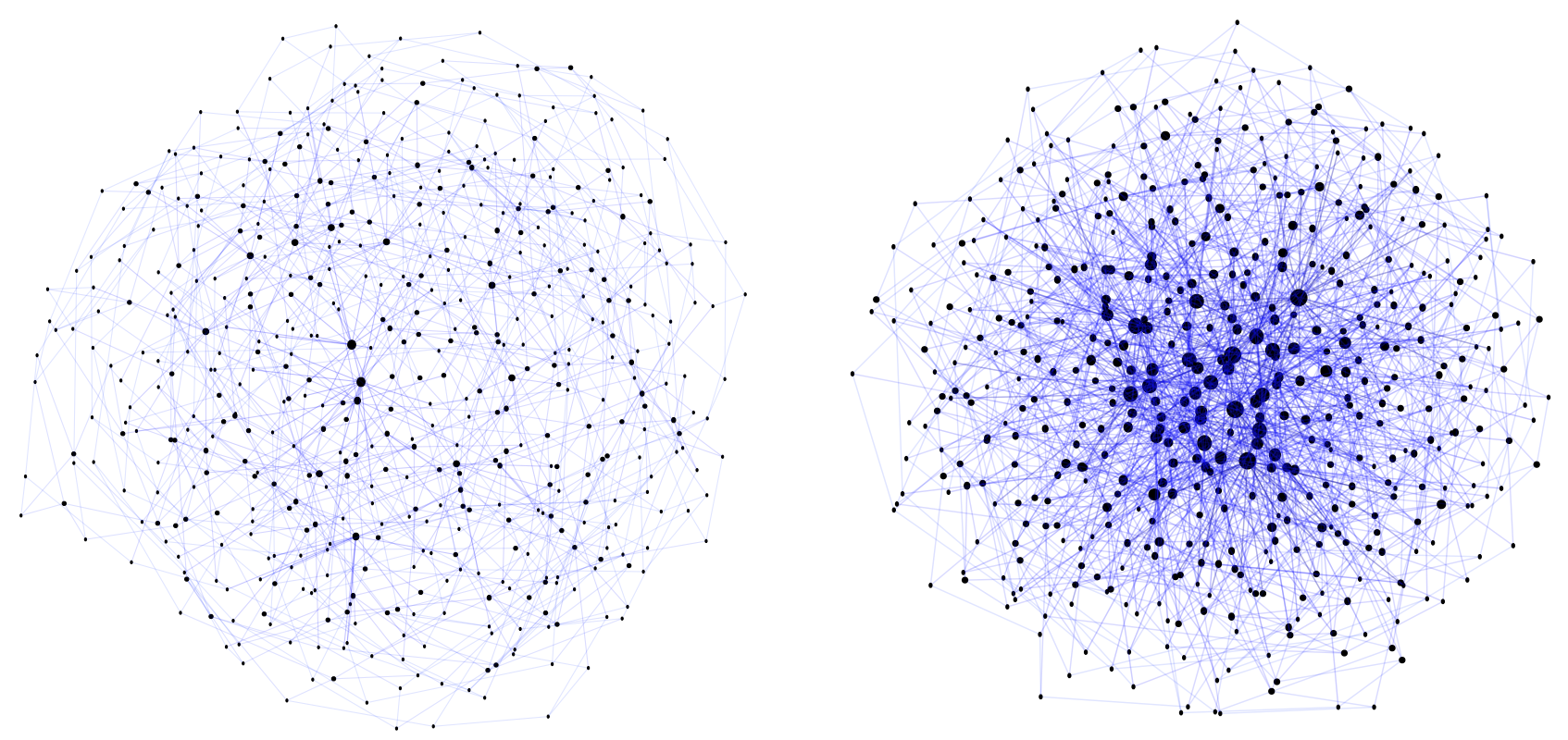

FIG. 1: (Color online) Typical networks with $N=512$ spins like those which were simulated. The left panel is for $\lambda=4.5$, where the network resembles a random graph. The right panel is for $\lambda=2.5$, at which value the network has similarities to many scale-free networks in nature and sociology $[15,16]$. The number of edges that each vertex (spin) has is encoded in the size of the dot. Large dots represent strongly-connected spins, whereas small dots have few connections. Note that we choose a minimum connectivity of 3 to prevent dangling ends in the network. As can be clearly seen, smaller values of $\lambda$ make for higher connectivity on a few nodes.

$\lambda=4.5$; however, there are only modest changes in the numbers of pure states seen. Finally, the number of pure states is decreased by the random field (and would be expected to be unity above the de Almeida-Thouless line [9], if any [14]).

The number of pure states when $H=0$ was shown to increase as $\sim N^{1 / 6}$ in Ref. [18] for the Sherrington-Kirkpatrick fully-connected model [20], and a similar $N$ dependence would be expected when $H \neq 0$. It is this slow $N$ dependence that is probably the source of the small number of pure states for the scale-free networks which are known to be mean-fieldlike [13].

Note that one should not confuse pure states with metastable states, such as the states that are simply stable against flipping just a single spin. States for which $S_{i} H_{i}>0$, $i=1,2, \ldots, N$ are exponentially numerous [21], rather than rare like pure states. Furthermore, pure states have free energies which differ only by $\mathcal{O}(1)$ from each other, while metastable states can have free energies which differ by $\mathcal{O}(N)$.

The existence of multiple pure states allows a choice: Any party which has existed for some time will probably already have a policy on some of the issues; i.e., some of their proposals could really be just minor variants on existing policies. Parties dislike having to change their policies, and when they do, they are typically mocked for doing so, e.g., for belatedly recognizing the error of their previous decisions. They could minimize this embarrassment by adopting the pure-state solution which has the greatest overlap with their existing policies, even if it were not the ground state. Note also, that the pure states correspond to portfolios of policies which have a high degree of internal consistency. It might be that they could be identified with the policies of each of the competing parties. In principle with $N$ issues, which a party might be in favor of or not, there exist $2^{N}$ positions on these policies, and one could envisage that $2^{N}$ parties might exist to represent all of them. In fact, in most countries the number of parties is small, which is just like the number of pure states, which is also small. Furthermore there is often a similarity in the policies of the various parties. For example, one speaks of parties as on the "right" or "left," which implies that some of them have similar policies, i.e., overlaps $q$. But this is just as would be expected if the pure states have an ultrametric topology [9].

Systematic procedures have been developed to identify pure states [22-24]. They essentially provide a way of determining the magnetization in the pure state $\alpha, m_{i}^{\alpha}=\left\langle S_{i}\right\rangle_{i}^{\alpha}$. Because this is obtained via a simulation at finite temperature, the last step would be to set $S_{i}^{\alpha}=\operatorname{sign}\left(m_{i}^{\alpha}\right)$, to identify the archetypal spin configuration associated with the pure state. We have not carried out the process of identifying the actual spin configurations of the pure states of the spin-glass models on scale-free networks above (other than that of the ground state) as it is computationally difficult, and we have just contented ourselves with showing that pure states exist for the models we have been simulating. Of course, when real data become available this work should be undertaken.

Spin-glass states are also chaotic [25]; i.e., a small change 

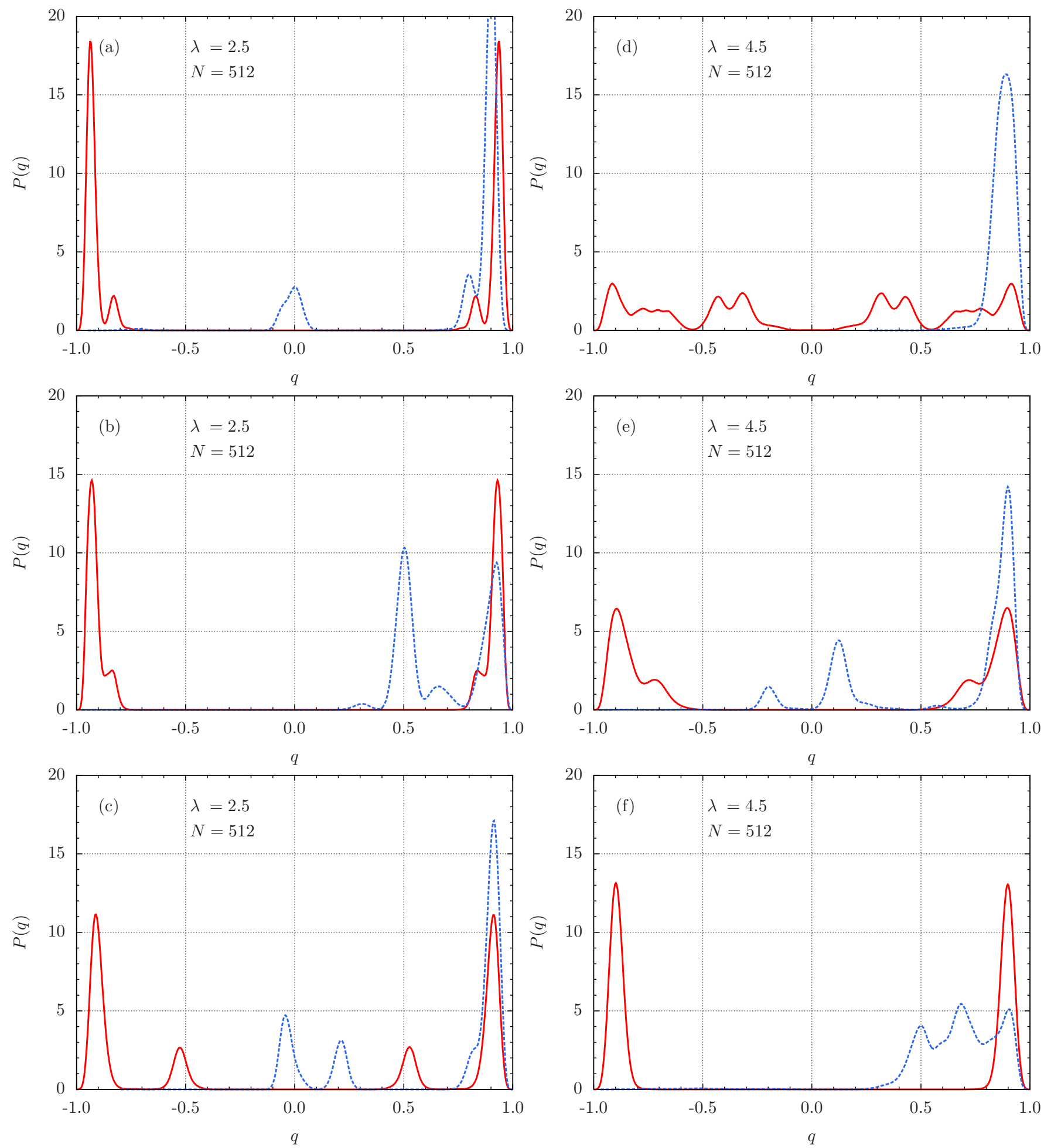

FIG. 2: (Color online) Example distributions $P(q)$ for $N=512$ and individual different interactions $J_{i j}$, as well as random fields $h_{i}$ on scale free networks with $\lambda=2.5$ (left) and $\lambda=4.5$ (right) and $T=0.2862 \ll T_{c}$. Solid lines (red) are for $H=0$, whereas dashed (blue) lines are for $H=0.10$. Note that when $H=0$ (solid curves) the distributions are symmetric, i.e., $P(q)=P(-q)$. In panels (a), (b), and (c) there seem to be 4 peaks/shoulders for both $H=0$ and $H=0.1$, while for panels (d), (e), and (f) 12 [1], 4 [6], and 2 [6] peaks are visible for $H=0[H=0.10]$. 
in the values of the interactions $J_{i j}$ or the fields $h_{i}$ can trigger a large change in the spin configurations of the pure states. However, that is the case in the thermodynamic limit when $N \rightarrow \infty$. For systems with $N$ values of the size of interest to us and defined on a scale-free mean-field-like topology, chaos will be quite hard to observe [26, 27]. This is fortunate as a party would like its policies to have stability against what might be just a passing whim of the electorate. The whims feed into changes in the $J_{i j}$ and $h_{i}$. Note that if Eq. (4) were used to determine the values of the $S_{i}$, a change of sign of any of the $h_{i}$ would result in a change in the $S_{i}$. However, using Eq. (10), that need not happen. Still, in general, if the couplings $J_{i j}$ change and the fields $h_{i}$ change sufficiently, the ground state orientations $S_{i}^{(T=0)}$ could be altered.

Finally, we have investigated the overlap $q^{\text {diff }}$ of the ground state configuration $S_{i}^{(T=0)}$ with

$$
\operatorname{sign}\left[\mathrm{m}_{\mathrm{i}}^{(0)}\right]=\operatorname{sign}\left(\mathrm{h}_{\mathrm{i}}\right)
$$

to show the extent of the differences between results obtained via the naive approach in Eq. (4) and that of Eq. (10), which includes the effects of correlations, to determine the policies which go into the manifesto:

$$
q^{\mathrm{diff}}=\frac{1}{N} \sum_{i=1}^{N} S_{i}^{T=0} \operatorname{sign}\left[\mathrm{m}_{\mathrm{i}}^{(0)}\right] .
$$

For $\lambda=2.5, N=512$, and $H=0.1$ we find after an average over 509 samples $q^{\text {diff }}=0.046(2)$, while for 510 samples and $\lambda=4.5, q^{\text {diff }}=0.052(2)$ [28]. In other words, the two different algorithms for choosing the policies for the manifesto result in spectacularly different manifestos (the value of their overlap $q^{\text {diff }}$ is close to zero)! We would, however, expect $q^{\text {diff }}$ to increase with increasing $H$.

\section{DISCUSSION}

A questionnaire containing a list of proposals could be constructed to find out whether there is a "gap in the market" for a new party. If there were four pure states generated, it would indicate there might, at least in the UK, be scope for an additional national-level party. Conversely, if there were only two pure states, it would suggest that a merger or coalition of the parties might be sensible.

One might wonder whether the procedures being used here could be also used for predicting the outcome of elections. In Ref. [6] the questionnaire is similar in its form to that outlined in Sec. I. There will also be correlations between the responses to the various questions. But in predicting the outcome of elections, one really needs only to ask individuals who they will vote for and whether they will actually be bothered to vote. The questionnaire in Ref. [6] is formulated so as to understand what factors and issues are influencing individuals to vote for a particular party and the circumstances of those who are choosing to support a particular party.

The spin-glass model could be of generic use whenever there exist correlations between choices: For example, a car manufacturer can produce models in a large number of variants, e.g., with or without a sun roof, with or without cruise control, with or without automatic transmission, with or without aluminum wheels, with or without leather upholstery, with or without fuzzy purple dice on the rear-view mirror, etc. Manufacturers have to decide what levels of trim they should send out to their dealers, and dealers have to believe that they can sell these examples. For well-established models they have past sales data to guide them as to what sells, but for a new model that information is lacking. There are correlations between customer choices. One factor dominates above all, price (just as the economy is thought to be one of the dominant factors in elections) [6, 7]. Dominance by just a few issues makes spin-glass behavior more pronounced $[12,14]$. Again, one could determine the desirability of the options with questionnaires and analyze the results with the aid of the spinglass model. It could be then that two or three levels of trim would emerge as pure states and manufacturers could send out to dealers models which correspond to these trim levels, thereby optimizing their sales.

\section{Acknowledgments}

We thank Professor Jane Green for drawing to our attention the useful website of Ref. [6] and Professor Ed Fieldhouse for other useful references, as well as Dr. M. Fischer for pointing us to smartvote.ch. We also thank R. S. Andrist for assistance with Fig. 1. M.A.M. thanks Alain Billoire for his collaboration in the early stages of this work. H.G.K. acknowledges support from the NSF (Grant No. DMR-1151387) and thanks Texas A\&M University for access to their Eos cluster.

\section{Appendix A: Illustrations of policies reduced to short sentences}

In this appendix we illustrate how policies can be reduced to short sentences suitable for assessment purposes via questionnaires. These examples are taken from the BBC 2010 Election website [5] from the heading "Crime." The full party manifestos are also available on Ref. [5]. These are the policies proposed by the three largest parties in UK politics. (It is common for news organizations in many countries to produce similar summaries at election times.)

\section{Conservative Party Policies}

$\square$ Replace police authorities with directly-elected police commissioners, with responsibility for strategy and budgets

Strengthen stop and search powers to tackle knife crime

Give police the power to publicly identify offenders

Change the law so that anyone acting "reasonably" to stop a crime or apprehend a criminal is not arrested or prosecuted 
Increase police and local authorities' powers to remove licenses from, or refuse to grant licenses to problem bars

Allow the police to use "instant sanctions" to deal with anti-social behavior, without criminalizing young people unnecessarily

Reduce paperwork needed for stop and search procedures

Increase prison capacity above Labour's plans, in order to scrap the early release scheme

Use private and voluntary sector groups to improve the rehabilitation of offenders, and pay providers by results

Allow courts to specify minimum and maximum sentences for certain offenders

Scrap ID cards and identity database.

\section{Labour Party Policies}

Protect "frontline" police from budget cuts in 20112013

Ensure that if a police forces fails consistently, either its chief constable will be replaced or it will be taken over by a neighboring force

Oppose elected police authorities or commissioners

"No-nonsense" one-to-one support for the 50,000 most "dysfunctional" families

Automatic parenting orders on those whose teenage children breach an ASBO (Anti-Social Behavior Order)

Guarantee an initial response to any complaint about anti-social behaviour within $24 \mathrm{~h}$ and give complainers a named case worker who will report back on progress
Make restorative justice available wherever victims approve it, bringing home to criminals the consequences of their crimes

Add 15,000 prison places by 2014

Give local people a vote on what community service offenders should do

Ensure that serious offenders are added to the DNA database "no matter where or when they were convicted"

Retain for six years the DNA profiles of those arrested but not convicted.

\section{Liberal Democrats Party Policies}

Increase police numbers by 3,000 over five years

Scrap identity card scheme

Make police authorities directly elected, with powers to sack and appoint the Chief Constable, set local policing priorities, and set budgets

Annual fitness tests for police officers

Replace filling out forms with new technology

Create a National Crime Reduction Agency to spread best practice through the force

Review police officers terms and conditions

Seek advice from Law Commission and Plain English Campaign to make paperwork more simple

Reduce the use of short sentences and encourage use of community sentencing to reduce prison overcrowding

Increase use of "restorative justice," forcing criminals to confront their behavior.
[1] S. Galam, Fragmentation versus stability in bimodal coalitions, Physica A 230, 174 (1996).

[2] S. Galam, Sociophysics: A Review of Galam Models, Int. J. Mod. Phys. C 19, 409 (2008).

[3] C. Castellano, S. Fortunato, and V. Loreto, Statistical physics of social dynamics, Rev. Mod. Phys. 81, 591 (2009).

[4] P. Contucci, E. Panizzi, F. Ricci-Tersenghi, and A. Sîrbu, A new dimension for democracy: egalitarianism in the rank aggregation problem (2014), (arXiv:physics.soc-ph/1406.7642).

[5] http://goo.gl/xxlH9V.

[6] http://bes2009-10.org.

[7] H. D. Clarke, A. Kornberg, T. J. Scotto, J. Reifler, D. Sanders, M. C. Stewart, and P. Whiteley, Yes we can! Valence politics and electoral choice in America, 2008, Electoral Studies 30, 450 (2011).

[8] D. Sanders, H. D. Clarke, M. C. Stewart, and P. Whiteley,
Downs, Stokes and the Dynamics of Electoral Choice, British Journal of Political Science 41, 287 (2011).

[9] M. Mézard, G. Parisi, and M. A. Virasoro, Spin Glass Theory and Beyond (World Scientific, Singapore, 1987).

[10] K. Binder, The Monte Carlo Method in Condensed Matter Physics (Springer-Verlag, Germany, 1995).

[11] D. P. Landau and K. Binder, A Guide to Monte Carlo Simulations in Statistical Physics (Cambridge University Press, 2000).

[12] D.-H. Kim, G. J. Rodgers, B. Kahng, and D. Kim, Spinglass phase transition on scale-free networks, Phys. Rev. E 71, 056115 (2005).

[13] H. G. Katzgraber, K. Janzen, and C. K. Thomas, Boolean decision problems with competing interactions on scale-free networks: Critical thermodynamics, Phys. Rev. E 86, 031116 (2012).

[14] Z. Zhu, J. C. Andresen, M. A. Moore, and H. G. Katzgraber, 
Boolean decision problems with competing interactions on scale-free networks: Equilibrium and nonequilibrium behavior in an external bias, Phys. Rev. E 89, 022118 (2014).

[15] A. L. Barabasi and R. Albert, Emergence of Scaling in Random Networks, Science 286, 509 (1999).

[16] A. Barabasi, H. Jeong, Z. Neda, E. Ravasz, A. Schubert, and T. Vicsek, Evolution of the social network of scientific collaborations, Physica A 311, 590 (2002).

[17] D. L. Stein and C. M. Newman, Spin Glasses and Complexity, Primers in Complex Systems (Princeton University Press, 2013).

[18] T. Aspelmeier, A. Billoire, E. Marinari, and M. A. Moore, Finite-size corrections in the Sherrington Kirkpatrick model, J. Phys. A: Math. Theor. 41, 324008 (2008).

[19] The simulations are done using the parallel tempering Monte Carlo method [29]. We use an optimized temperature set of 45 temperatures in the range $T_{\min }=0.2862$ and $T_{\max }=2.0$ that reaches deep into the spin-glass state. Equilibration is tested by the method outlined in detail in Refs. [13, 30, 31]. For all values of $\lambda$ and $H$ simulated we equilibrate for $2^{25}$ Monte Carlo sweeps and measure over the same amount of Monte Carlo time.

[20] D. Sherrington and S. Kirkpatrick, Solvable model of a spin glass, Phys. Rev. Lett. 35, 1792 (1975).

[21] A. J. Bray and M. A. Moore, Metastable states in spin glasses with short-ranged interactions, J. Phys. C 14, 1313 (1981).

[22] E. Domany, G. Hed, M. Palassini, and A. P. Young, State Hierarchy Induced by Correlated Spin Domains in short range spin glasses, Phys. Rev. B 64, 224406 (2001).

[23] E. Marinari, O. C. Martin, and F. Zuliani, Equilibrium valleys in spin glasses at low temperature, Phys. Rev. B 64, 184413 (2001).

[24] H. G. Katzgraber and A. K. Hartmann, Ultrametricity and Clustering of States in Spin Glasses: A One-Dimensional View, Phys. Rev. Lett. 102, 037207 (2009).

[25] A. J. Bray and M. A. Moore, Chaotic Nature of the Spin-Glass Phase, Phys. Rev. Lett. 58, 57 (1987).

[26] A. Billoire and E. Marinari, Overlap among states at different temperatures in the SK model, Europhys. Lett. 60, 775 (2002).

[27] T. Rizzo and A. Crisanti, Chaos in Temperature in the
Sherrington-Kirkpatrick Model, Phys. Rev. Lett. 90, 137201 (2003).

[28] No exact algorithms exist to determine the ground-state configurations of Ising spin glasses on scale-free networks. We use the method pioneered in Refs. [32] and [33] where a finite-temperature parallel-tempering Monte Carlo simulation is pushed to temperatures much smaller than the critical temperature. Two copies of the system are simulated in parallel. After a long thermalization time, the lowest energy states for each copy are recorded for a given measurement time. If after the end of this measurement time both lowest energies agree, we believe we have found the ground state of the system with high confidence. We use the same temperature set used for the determination of the overlap distributions [19]; however, we thermalize for $2^{26}$ Monte Carlo sweeps and perform the measurement for the same amount of time. For each combination of fields and $\lambda$, approximately 500 disorder instances have been simulated. For all zero-field simulations we are certain that the ground-state configurations have been obtained for all samples. For the finite-field simulations at $H=0.1$ less than $1 \%$ of the instances did not reach the lowest state. However, this should not bias the arguments presented in this paper.

[29] K. Hukushima and K. Nemoto, Exchange Monte Carlo method and application to spin glass simulations, J. Phys. Soc. Jpn. 65, 1604 (1996).

[30] H. G. Katzgraber, M. Palassini, and A. P. Young, Monte Carlo simulations of spin glasses at low temperatures, Phys. Rev. B 63, 184422 (2001).

[31] Z. Zhu, J. C. Andresen, M. A. Moore, and H. G. Katzgraber, Boolean decision problems with competing interactions on scale-free networks: Equilibrium and nonequilibrium behavior in an external bias (2013), (arXiv:cond-mat/1310.1139).

[32] H. G. Katzgraber and A. P. Young, Geometry of large-scale lowenergy excitations in the one-dimensional Ising spin glass with power-law interactions, Phys. Rev. B 68, 224408 (2003).

[33] H. G. Katzgraber, M. Körner, F. Liers, M. Jünger, and A. K. Hartmann, Universality-class dependence of energy distributions in spin glasses, Phys. Rev. B 72, 094421 (2005). 